\title{
Multicriteria Decision Analysis and Conversational Agents for children with autism
}

\author{
Micol Spitale \\ Politecnico di Milano \\ IBM Italy \\ micol.spitale@polimi.it
}

\author{
Fabio Catania \\ Politecnico di Milano \\ fabio.catania@polimi.it
}

\begin{abstract}
Conversational agents has emerged as a new means of communication and social skills training for children with autism spectrum disorders (ASD), encouraging academia, industry, and therapeutic centres to investigate it further. This paper aims to develop a methodological framework based on Multicriteria Decision Analysis (MCDA) to identify "the best", i.e. the most effective, conversational agent for this target group. To our knowledge, it is the first time the MCDA is applied to this specific domain. Our contribution is twofold: i) our method is an extension of traditional MCDA and we exemplify how to apply it to decision making process related to CA for person with autism: a methodological result that would be adopted for a broader range of technologies for person with impairments similar to ASD; ii) our results, based on the above mentioned method, suggest that Embodied Conversational Agent is most appropriate conversational technology to interact with children with ASD.
\end{abstract}

\section{Introduction}

In the last decades, many efforts have been made to investigate how assisted computer-based technology and social robots can help children with autism enhance their social skills through dialogue. Autism Spectrum Disorder (ASD) is a development disorder characterized by communication impairments and repetitive behaviors. Individuals with autism have problems in social interactions because of their deficits in language, communication, emotion expression and sensory impairments [1]. In [2], [3], [4], [5] and [6], authors underline the power of children computer-based interactions with respect to the interaction with other people for three main reasons:

- computers environment are predictable and consistent
Pietro Crovari

Politecnico di Milano

pietro.crovari@polimi.it

- children can work according to their social skills level, because computer-based system are flexible and customized

- each assigned task can be repeated until the goal is reached and children can be rewarded with real-time feedback

Therefore keeping in mind users' needs, innovative interactive technologies could help them improve their abilities and further skills, such as communicative and social ones. Since children with autism are characterized by unpredictable behaviour and uniqueness, we must be aware that each child has their own attitude and capability. For this reason, it is crucial to provide a system which fulfills user requirements in the more flexible and customized way. We opted to investigate the adoption of conversational technologies to enhance communication skills of children with ASD, such as ability to comprehend, detect or apply aural conversation to engage in discourse effectively with others, and improve their social capabilities. To properly engage and capture attention during speech interaction, the voice-based device should convey a natural dialogue and keep them focus during the conversation. The conversational technologies can lead to different interaction channels, such as computer or tablet-based, robot-based, and only voice-based conversational technologies.

Starting from that, we analyzed how different speech-based interaction technologies are affecting the performance and perception during children-agent speech interaction [7]. The question we would like to answer is: 'Which is the most appropriate conversational agents for interacting with children with autism?'. The use of the Multi-Attribute Value Theory (MAVT) in decision problems related to interaction technologies is not an easy task. We explore the use of a Multi-Attribute Value Theory (MAVT) method to address this problem in the conversational technologies domain because it is characterized by conflicting objectives and different actors involved. MAVT is a 
particular Multicriteria Decision Analysis (MCDA [8], [9]) technique, and it can be used to address problems that involve a finite and discrete set of alternative actions that have to be evaluated on the basis of conflicting objectives. For any given objective, one or more different attributes or criteria are used to measure the performance in relation to that objective.

MAVT was applied to this problem, where a decision was made regarding the adoption of the most appropriate conversational agent to interact with children with autism. We illustrate the motivation and the challenges behind this ambitious project, shedding a light on the potential of conversational agents and their adoption with children with autism through a MCDA approach. Besides the fact that it is the first time that decision analysis is applied to this innovative case study, the contribution brought by this research refers to: i) the methodological approach which merges framing, value focused thinking tools with MCDA; ii) identification of the most appropriate conversational agent to interact with children with ASD. This work has an innovative value because it sheds a light on the possibility to exploit decision making analyses to support researchers or enterprises analyzing in depth the adoption of one conversational technology with respect to another to support every-day life tasks for children with ASD.

The paper is divided as follows: in the first part we analyze the related work concerning the conversational agents applied in interaction with ASD children to give the reader the possibility to fully understand the problem. Then we describe the methodology used during the decision process, and after that we discuss the results obtained from the study, focusing on the contribution of this method for supporting the decision process.

\section{Background: Conversational Agents for children with autism}

Many authors dealt with conversational agent-human interaction from different perspectives. In literature, authors divide into two sides: on one hand, researchers focused on virtual agents, which are tutors on tablet or computer screens with abstract, cartoon-like or human-like appearance, called Embodied Conversational Agents (ECA) to assist children with neurodevelopment disorders (broader term which includes also autism spectrum disorders), while on the other hand many authors deeply investigated the efficiency of physical conversational agents, names as Social Robots, in training social skills of children with autism. In literature, both ECA and speaking Social Robots have been investigated to assist children with autism.

In learning domain since 1999, Cassel [12] argues that ECA can be exploited to support student. Tartaro and Cassel [13] discussed the intervention of a social peer for children with conversational impairment, proposing different solutions during years such as SARA [11] (Socially Aware Robot Assistant), which relies on information about human-virtual assistant relationship, Alex, [14] which aims at improving achievement in multicultural classrooms, RAPT [15] (Rapport- Aware Peer Tutor) a virtual peer tutor designed to build relation and support student learning, and Sam, an authoring agent for supporting social interaction skills and scaffolding literature skills [16]. Also Milne, Marissa, et al. [17] provided a ECA as a tutor to improve social competence in children with autism, since ECA is capable to present content in a way that suits different learning style and sensory needs. Another important contribution is given by Rachel, an ECA designed to elicit and analyze interactions and encourage affective and social behavior for children with ASD [18]. They designed an emotionally targeted interactive agent for children with autism based on Wizard-of-Oz to provide an effective platform for eliciting and analyzing children communicative abilities. Analogously, [6] authors developed a multi-modal automated embodied conversational agent for training social skills to prove its efficiency with children with ASD.

In the last years, researchers started to investigate how speech based interaction is perceived by the user. In [19], authors specifically focused on how children interact with off-the-shelf speech agents, conducting an exploratory study using different conversational agents such as Google Home, Alexa or Cozmo. The outcome of this study is that children perceived virtual assistant not as smart as they are. Finally, Williams et al. [20] argued that children are comfort to talk with a doll.

Other authors focused on Social Robots related to people with ASD (e.g., [21], [22], [23], [24]). Many social robots used in ASD therapy are remotely controlled by caregivers [22], [24], and authors showed how powerful was the interaction with them even if only non-verbal behaviours were performed. They have been used successfully to attract attention, stimulate imitation, and improve communication, socialization, and behavioral skills needed for independent living. Several researches explore the shape and movement capability of robots in relationship to subjects with ASD. Different shapes have been explored, from abstract ones to cartoon-like, simplified humanoids, or realistic human-like faces [21]. Results pinpoint that individuals with ASD show a preference for something 

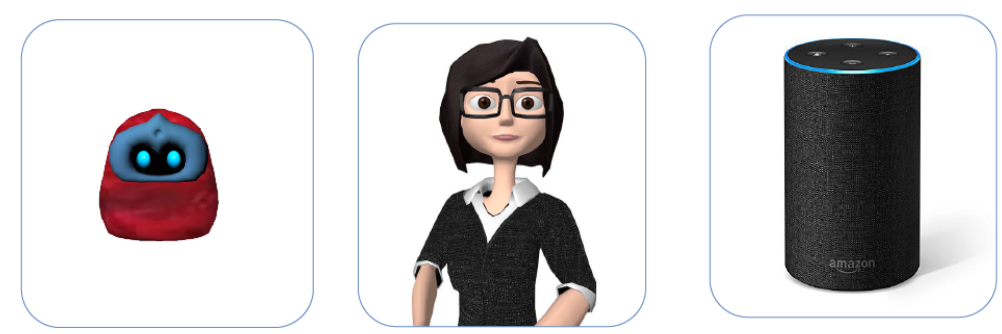

Figure 1. Alternatives: Social robot (a)[10], Embodied Conversational Agent (ECA) (b)[11], Virtual assistant (c)

that is clearly artificial compared to agents that have human-inspired characteristics [25]. Some authors suggest that the shape of the robot should evoke a familiar element, such as a toy that the subject likes, or a cartoon character. Several social robots exploit emotional features that seem to benefit children with ASD. Keepon [24] is a creature-like robot that is only capable of expressing its attention (directing its gaze) and emotions (pleasure and excitement). An empirical study with autistic children showed that the robot triggered a sense of curiosity and security, and the subjects spontaneously approached Keepon and engaged in dyadic interaction with it, which then extended to triadic interactions where they exchanged with adult caregivers the pleasure and the surprise they found in Keepon. KISMET [26] is an emotional robot which possesses eyebrows, ears, and a mouth and expresses emotions depending on the way a human interacts with the robot. The robot's emotional behavior is designed to generate an analogous social interaction to a robot-human dyad as for an infant-caretaker dyad.

Besides these great contributions, the research has been divided for a long time. It urges to find a methodology to allow young researchers and industries to clearly frame the problem and take the right research path or invest money for social goods, according to real effect on users with autism.

\section{Methodology}

To understand which is the most effective agent to talk with people with ASD, we opted for the Multicriteria Decision Analysis (MCDA) approach because it is a valuable and widely-used tool to support decision making process where there is a choice to be made between discrete and finite options. This method is usually exploited where several issues such as social and economical ones are involved and shall be taken into account during process. Moreover, it is used when the majority of the objectives are conflicting, requiring the decision maker (DM) to manage them and make trade-offs [27], [28], [29]. The subsequent process to build a MAVT consists on the following steps: (i) framing the problem, (ii) identifying objectives and designing alternatives, (iii) assessing score for each alternative, (iv) defining value functions associated to each attribute of the objectives and finally (v) ranking the alternatives. In the following sections, these steps will be examined.

\subsection{Framing}

As a first step, we set the problem, analyzing purpose, perspectives and scope according to [30].

Purpose. We have different conversational technologies to help patients and this gives the possibility to develop a method to compare them. We want to find a tool to improve daily life of children with autism and help people that support children. We would like to increase improve the accessibility of technologies during therapy. Monitoring the improvements in short term period could help more and more caregivers adopt conversational technologies in therapeutic centre.

Perspectives. We would like to explore the problem from different perspectives analyzing who should be involved in this process: not only children with ASD are involved, but also their parents and therapists.

Scope. As a hypothesis, only the current existing technologies are taken into account. At a first step, we focus on feasibility, cost, accessibility, and child engagement; while later we will determine the application scenarios (i.e. institution or/and home) and the choice of enterprise involved in decision process.

The alternatives analyzed for answering our main question ('Which is the most appropriate conversational agent for interacting with children with autism?') are existing conversational technologies:

- Social robots (Fig.1.a [10]) 


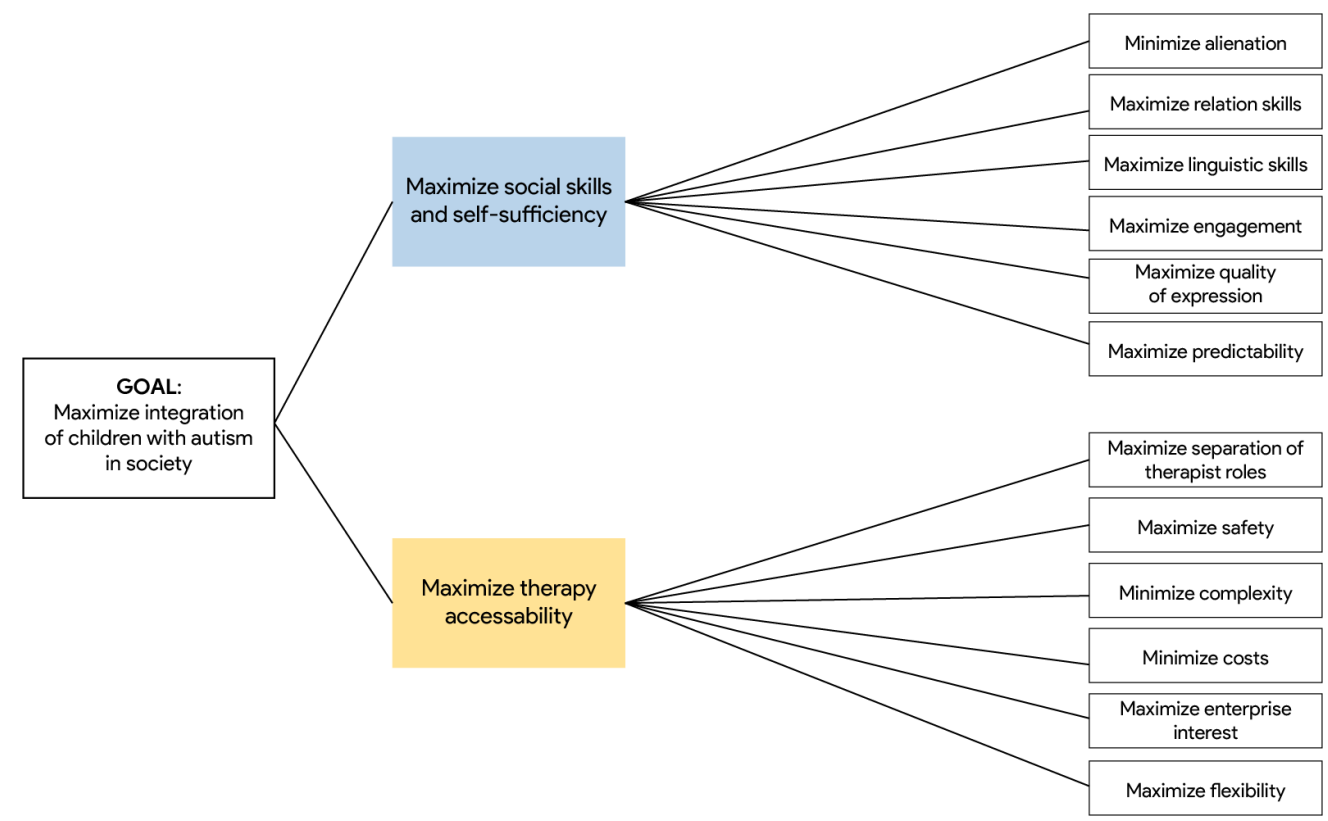

Figure 2. Value tree: the outcome of the value-focused thinking

- Embodied Conversational Agent (Fig.1.b [11])

- Virtual assistant (i.e. Google Home, Alexa) (Fig.1.c)

In addition, we introduces also the Human alternative, just to compare and analyze all different possible solutions for increasing social skills, self-sufficiency, and therapy accessibility through conversational interaction.

\subsection{Objectives}

Objectives are 'statements of something that one desires to achieve' [31], thanks to the interaction between the child and the agent, and they depend on several aspects, such as the actors involved in the decision process. It is crucial to take into account also the environment in which the decision process is taking place. Through brainstorming, our team composed by decision analysts and Decision Maker (DM) arrived at a common list of objectives that allows us to recognize a first list with an immediate approach. The twelve objectives that we were satisfied with are: engagement, performance, effectiveness, cost, customization, configurable, reliability, assessment of progress, predictability, adaptability, reverse inclusion, accessibility. As researchers underline [32], when decision maker (DM) and decision analysts have to think about objectives, they omit half of the objectives, missing the most relevant ones. For this reason, we decided to adopt value-focused thinking approach, exploiting techniques for enlarging our objectives list [31], [33]. By applying the value-focused thinking method, we extended the list of objectives to twenty ( $67 \%$ more) and we formalized them as follows:

1 Maximize engagement and attention

2 Maximize therapy accessibility

3 Maximize communication performance of the children

4 Minimize the complexity of use to allow programming by therapist

5 Maximize flexibility to adapt to different patients

6 Minimize bad reaction and frustration of children

7 Maximize safety during therapeutic session

8 Minimize scaring effects

9 Maximize the affection

10 Minimize the alienation

11 Minimize the cost

12 Maximize the predictability

13 Maximize the quality of the expression of the agent

14 Minimize the human influence and impact on automatic tasks 


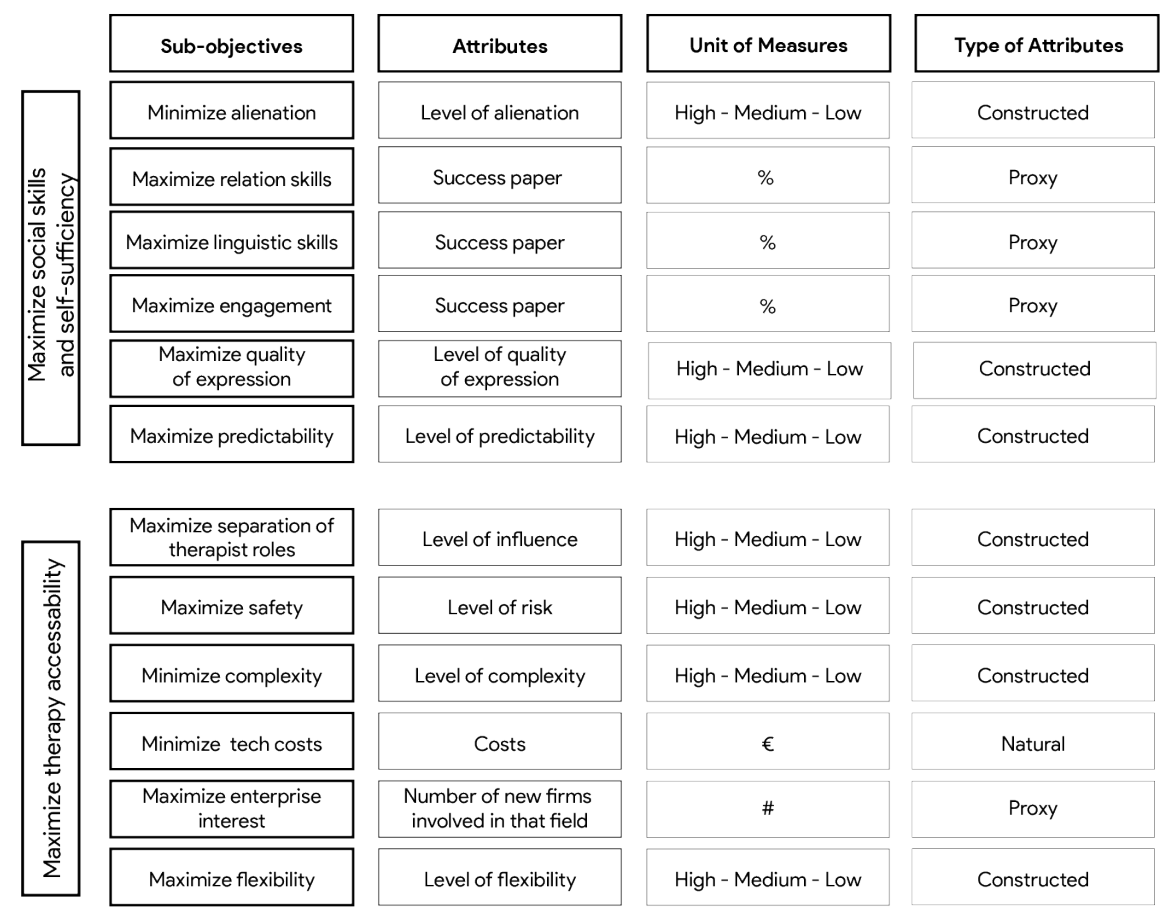

Figure 3. Objectives and attributes: for each sub-objective an attribute is defined with a corresponding unit of measure

15 Maximize the separation of therapeutic role

16 Maximize the independence of children

17 Maximize the social image of enterprise

18 Minimize the addiction

19 Maximize the social skills

20 Maximize the integration of patients in the society

\subsection{Value tree and attributes definition}

Once twenty objectives were elicited, we clustered and filtered them applying the value-focused thinking and constructing the value-tree. The value-tree is useful to understand that not all of the objectives are on the same level, classifying them in different levels. Indeed, we came up with two different layers of objectives: one layer refers to the two major individuated categories and the second one contains the corresponding sub-objectives. We arrived to twelve objectives, divided into two main groups of six objectives each. We used a top-bottom approach by starting from the most general objective and by asking 'how can we reach this?'. This approach helped us also to individuate repetitive objectives (e.g. same unit of measurements). The final outcome of the value-based focus thinking is the value tree depicted in Fig.2.

The degree to which objectives are achieved is measured through a set of attributes, which may be natural (they follow directly from the definition of the objective), constructed (they specify a finite number of degrees to which objectives are met), and proxy (they are only indirectly linked to the definition of the objective) [31]. In Fig. 3, we collected all the attributes assigned to each objectives.

We list the constructed attributes of objectives with their definitions [34], [35]:

- Level of alienation. Low: Child reacts always to external inputs during interaction with the agent; Medium: Child reacts often to external inputs during interaction with the agent; High: Child reacts seldom or never to external inputs during interaction with the agent.

- Level of quality of expression. Low: Artificial voice, non-expressive and monotonic voice; Medium: Natural, but not expressive voice (recorded-like); High: Natural and expressive voice.

- Level of predictability. Low: Unpredictable action, situation-dependent action (movement-questions, etc); Medium: Partially 

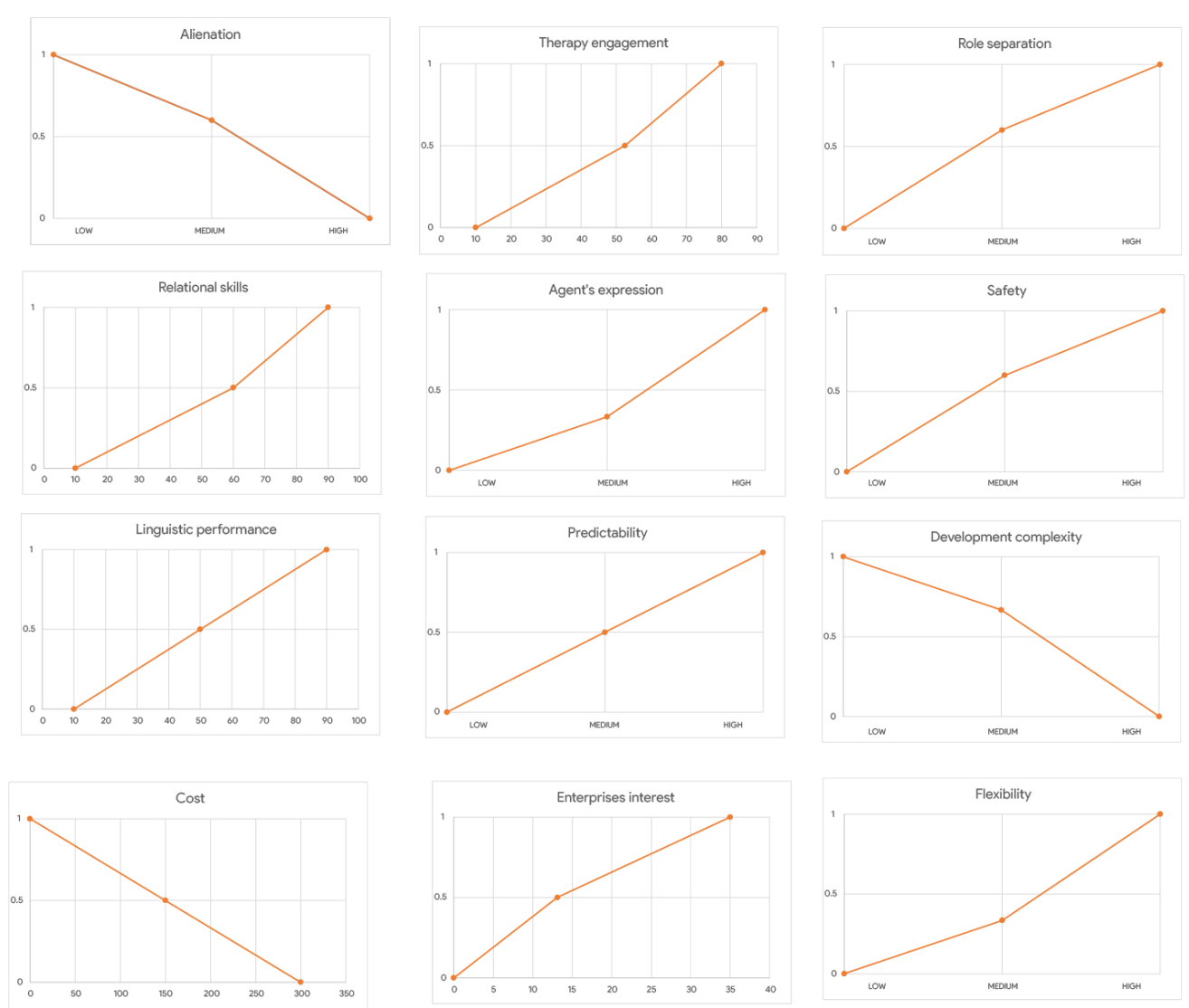

Figure 4. Value functions: functions applied to transform performance matrix to evaluation matrix

unpredictable actions (ex. Predicted questions, but unpredicted /sudden movements); High: Totally predictable actions.

- Level of influence. Low: Human completely involved in both (automatic/relational); Medium: Human partially involved in automatic tasks; High: Human dedicated only to relational tasks.

- Level of risk. Low: May cause medical condition and/or physical damage; Medium: Minor injuries; High: Completely safe.

- Level of complexity. Low: No software implementation or very simple implementation required; Medium: Moderate software implementation (small effort); High: High effort for software implementation.

- Level of flexibility. Low: No capability to adapt to the situation; Medium: Limited capability of adaptation to the situation (ex. Movements or appearance); High: Full adaptability (ex. Human behavior).
The proxy attributes Success Paper refers to the number of paper which successfully provide evidence on efficient of conversation technologies with children with ASD.

\subsection{From performance matrix to the evaluation matrix}

In this step the performances of each alternative were specified for each attribute. In some cases, the performances were readily available, in some other cases they had to be computed or estimated ad hoc for the problem at hand [36]. We then focused on the winner for each objective and we observed that neither a decoy option nor winner was detected.

Then we defined different value functions for each objective: on one hand we used the bisection elicitation process for the quantitative attributes, and on the other hand we exploited the direct elicitation for the constructed ones. The direct rating technique consists of the DM estimating the strengths of preferences for different levels of attributes, while the bisection method consists of identifying midpoints of value given two extreme levels within the range of attributes [36]. Once 


\begin{tabular}{|c|c|c|c|c|}
\hline & $\begin{array}{c}\text { Embodied } \\
\text { Conversational Agent }\end{array}$ & Social Robot & Virtual Assistant & Human \\
\hline $\begin{array}{c}\downarrow \text { Alienation } \\
\uparrow \text { Relational skills }\end{array}$ & 0 & 0 & 0.6 & 1 \\
$\uparrow$ Linguistic performance & 0.667 & 1 & 0 & 0.4 \\
$\uparrow$ Therapy engagement & 1 & 0.625 & 0 & 0.5 \\
$\uparrow$ Quality of agent's expression & 0.818 & 1 & 0 & 0.636 \\
$\uparrow$ Predictability & 0.333 & 0.333 & 0 & 0 \\
$\uparrow$ Therapist's role separation & 1 & 0.5 & 0.6 & 0 \\
$\uparrow$ Safety & 1 & 0.6 & 0.6 & 1 \\
$\downarrow$ Development complexity & 0 & 0 & 1 & 1 \\
$\downarrow$ Technology cost & 0.667 & 0 & 0.5 & 1 \\
$\uparrow$ New enterprises potential interest & 0.933 & 0 & 0.291 & 0 \\
$\uparrow$ Flexibility & 1 & 0.84 & 0 & 1 \\
\hline
\end{tabular}

Figure 5. Evaluation Matrix: the up-arrow refers to maximize objective while the down-arrow indicate the minimization of the corresponding objective

the value functions have been defined as depicted in Fig. 4 , we applied them to the performance matrix and the result was the evaluation matrix shown in Fig. 5,

\subsection{Weighting}

The weights of each objectives were elicited adopting the swing-weights technique, which is based on the direct analysis of the value advantage associated with swinging between the end point of each attribute range [37]. Using the swing method, we asked the Decision Maker (DM) several questions, one for each attribute. First of all, we asked DM to imagine to be in a situation where all of one's attributes are at their worst level and to choose which one to improve and swing. So DM put 100 points (over 100) to the chosen one. Then, DM repeated the reasoning with the remaining attributes and chose another to improve. Reasoning on the fact that the first swing was worth 100 points, DM relatively decided how many points it would give to the second (for example 70 points). The process continued repeating the same reasoning for all attributes of sub-objectives and then we weighted also the corresponding category. The resulting weights are collected in Fig. 6 . On one hand, the main advantages of this technique are that DM should only know the attributes range and not the value functions associated to them, so that the elicitation of weights is independent from the function's shape. On the other hand, this technique doesn't include consistency checks, so the outcomes cannot coincide with realistic scenario alternatives.

Since we had two different levels in the value tree, we gave weight to the lower level of sub-objectives and then to the upper one (Maximize social skills and self-sufficiency and Maximize therapy accessibility) using additive models [38]. This means that the overall value of alternatives is computed as the linear combination of weights and attribute values of each objective.

\section{Results and Discussion}

\begin{tabular}{ccc}
\hline Ranking & Alternatives & Scores \\
\hline First & ECA & 0.654 \\
Second & Human & 0.584 \\
Third & Social Robot & 0.514 \\
Fourth & Virtual Assistant & 0.441 \\
\hline
\end{tabular}

Table 1. Final ranking of alternatives

\subsection{Results and sensitivity analysis}

Once the decision process analysis have been concluded, the alternatives were ranked. The Embodied Conversational Agent (ECA) is the winner after the first iteration as depicted in Tab. 1. Although the Human has the largest number of best-scoring objectives, the ECA has high scores in the objectives that are weighted the most. The last step consists on testing the stability of the results. For this sensitive approach, we selected four scenarios to analyze running a Monte Carlo Simulation. Firstly, we chose the first as the one analyzed in the previous sections as the baseline. Then, we examined balanced weights by setting all of the weights to 1 and then normalizing them. As a second scenario, we choose an analysis on stochastic variation of primary objectives, represented in Tab. 2. The weights of the first level objectives were randomly generated while the weights for the secondary objectives were set equal to the baseline case. After that, we investigated the stochastic variation of secondary objectives, randomly generating the secondary objectives and fixing the primary ones to the baseline values, as shown in Tab. 3. As final consideration, we proceeded with a stochastic variation analysis of all objectives randomly, depicted in 4 . Since the ECA has high scores in the objectives that are 


\begin{tabular}{|c|c|c|c|}
\hline & Swing-weights & Normalized weight & Weight categories \\
\hline \multirow{6}{*}{$\begin{array}{c}\downarrow \text { Alienation } \\
\uparrow \text { Relational skills } \\
\uparrow \text { Linguistic performance } \\
\uparrow \text { Therapy engagement } \\
\uparrow \text { Quality of agent's expression } \\
\uparrow \text { Predictability }\end{array}$} & 70 & 0.167 & 0.098 \\
\hline & 100 & 0.238 & 0.140 \\
\hline & 60 & 0.143 & 0.084 \\
\hline & 80 & 0.190 & 0.112 \\
\hline & 50 & 0.119 & 0.070 \\
\hline & 60 & 0.143 & 0.084 \\
\hline TOT & 420 & & \\
\hline \multirow{6}{*}{$\begin{array}{c}\uparrow \text { Therapist's role separation } \\
\uparrow \text { Safety } \\
\downarrow \text { Development complexity } \\
\downarrow \text { Technology cost } \\
\uparrow \text { New enterprises potential interest } \\
\uparrow \text { Flexibility } \\
\end{array}$} & 100 & 0.222 & 0.092 \\
\hline & 60 & 0.133 & 0.055 \\
\hline & 70 & 0.156 & 0.064 \\
\hline & 50 & 0.111 & 0.046 \\
\hline & 80 & 0.178 & 0.073 \\
\hline & 90 & 0.200 & 0.082 \\
\hline TOT & 450 & & \\
\hline
\end{tabular}

\begin{tabular}{|c|c|c|}
\hline & Swing-weights & $\%$ \\
\hline Max. social skills and self-sufficiency & 100 & 0.588 \\
Maximize therapy accessability & 70 & 0.412 \\
\hline TOT & \multicolumn{2}{|c|}{170} \\
\cline { 2 - 3 }
\end{tabular}

Figure 6. Weighting: the first table collects the weights of the sub-objectives, while the second collects the weights corresponding to the categories (the up-arrow refers to maximize objective while the down-arrow indicate the minimization of the corresponding objective)

weighted the most, the sensitivity analysis has shown a low stability of the results.

\begin{tabular}{ccccc}
\hline & First & Second & Third & Fourth \\
\hline ECA & $100 \%$ & $0 \%$ & $0 \%$ & $0 \%$ \\
Social Robot & $0 \%$ & $10.5 \%$ & $72.4 \%$ & $17.1 \%$ \\
Virtual Assistant & $0 \%$ & $0 \%$ & $17.1 \%$ & $82.9 \%$ \\
Human & $0 \%$ & $89.5 \%$ & $10.5 \%$ & $0 \%$ \\
\hline
\end{tabular}

Table 2. Stochastic of primary objectives

\begin{tabular}{ccccc}
\hline & First & Second & Third & Fourth \\
\hline ECA & $56.9 \%$ & $42.8 \%$ & $0.3 \%$ & $0 \%$ \\
Social Robot & $0 \%$ & $9.5 \%$ & $72.1 \%$ & $18.3 \%$ \\
Virtual Assistant & $0 \%$ & $0.9 \%$ & $18.1 \%$ & $81 \%$ \\
Human & $43.1 \%$ & $46.8 \%$ & $9.5 \%$ & $0.7 \%$ \\
\hline
\end{tabular}

Table 3. Stochastic of secondary objectives

\begin{tabular}{ccccc}
\hline & First & Second & Third & Fourth \\
\hline ECA & $55.4 \%$ & $42.7 \%$ & $1.9 \%$ & $0 \%$ \\
Social Robot & $0.6 \%$ & $11.2 \%$ & $50.5 \%$ & $37.8 \%$ \\
Virtual Assistant & $0 \%$ & $2.5 \%$ & $36.2 \%$ & $61.3 \%$ \\
Human & $44 \%$ & $43.7 \%$ & $11.4 \%$ & $0.9 \%$ \\
\hline
\end{tabular}

Table 4. Stochastic of all objectives

\subsection{Discussion}

Exploiting the Multicriteria Decision Analysis (MCDA) approach, we suggest which is the most effective conversational agents to interact with children with Autism Spectrum Disorders (ASD). The results show that Embodied Conversational Agents (ECA) are the most suitable conversational technology because they fulfill the most important objectives. What makes the ECAs the "best" conversational technologies for children with autism stays in:

- helping them in minimizing alienation;

- supporting them to maximize relational skills;

- enhancing therapy engagement;

- helping in separating automatic and relational tasks during therapy.

Besides the specific suggestion for the case study considered, we highlight the contributes in terms of decision making process. First of all by applying value-focused thinking method, we extended the list of objectives from twelve to twenty ( $67 \%$ more). Besides their quantities, we noticed that the new list had a series of advantages compared to the first one generating during the brainstorming phase. The new list of objectives contains our main goal and an objective related to a stakeholder that was not previously considered. Then some of the objectives of the first list were split into more specific ones. Moreover, the new objectives are more clear, differentiating between maximization and minimization. The first set of questions, regarding the list of what we want to achieve which can be found in [32], generate the greater number of objectives. However, they were all already included in the first list. New objectives 
have been generated by question of the good or bad scenario of alternatives (four new objectives), while questions related to goals, constraints, and guidelines to different perspectives and strategic objectives to the generation of two new objectives each. Once the evaluation matrix has been generated and the weights were assigned, it was clear that the most relevant objectives were missed in the first list generated during brainstorming step: just two of four of the most important objectives were elicited at the beginning of the process. This provided an evidence of how much exploiting the value-focused thinking based technique impacted the whole decision process. Merging the framing approach and value-focused thinking based method largely affected the decision analysis.

From sensitivity analysis, we found how many of the results were of low stability. Tab. 2 highlights the stable results for the sensitivity analysis associated with primary objectives, Tab. 3 and 4 show how much of the ranking of alternatives could change according to the assigned weights. This behaviour derives from the large number of attributed constructed of the objectives.

\section{Conclusion and future works}

The paper shows its innovative approach twofold: the use of Multicriteria Decision Analysis for a technology application in a social context, with a methodological approach, which merges the framing and a process boost with the exploitation of value focused thinking devices, and the results suggest that Embodied Conversational Agents (ECAs) are the most effective agents for children with autism to interact with. First of all, since the literature is divided, it would be important to provide this methodology as a tool to both young researchers and enterprises that want to invest in technologies for social good to better make their own decisions. With this paper, we want to provide a decision framework highlighting the power and impact of its exploitation in a decision analysis process. This paper gives some basic guidelines to properly analyze the adoption problem of conversational agents which are able to dialogue with children with autism.

There are many limitations of the study. Firstly, data is based on the decision maker expertise. Secondly, there is an unbalanced number of papers for the different technologies, which affect the proxy attributes (Success Paper [\%]). Furthermore, we defined only three levels for constructed attributes of objectives because of the great effort in eliciting them: with a more deep analysis it would be possible to enlarge the quality scale to at least five levels to be more accurate. Indeed, sensitivity showed a low stability of the nominal ranking, possibly due to the high number of constructed attributes of the objectives. However, the innovative approach of merging framing with value-based thinking allowed us to re-frame the problem and enriched objectives list, improving the decision analysis.

The described method could be exploited not only by young researchers to examine and frame their research properly but also by enterprises to give them the right direction to invest money in social goods. Further research could address the problem of constructed attributes, which definitions could be expanded also based on empirical studies, or expand the model to more stakeholders such as experts in the therapeutic and enterprise domains.

\section{Acknowledgments}

This research is supported by IBM Italia and EIT Digital, under Project Cognitive Computing for Cognitive Disability.

\section{References}

[1] I. Rapin and R. F. Tuchman, "Autism: definition, neurobiology, screening, diagnosis," Pediatric Clinics of North America, vol. 55, no. 5, pp. 1129-1146, 2008.

[2] C. Putnam and L. Chong, "Software and technologies designed for people with autism: what do users want?" in Proceedings of the 10th international ACM SIGACCESS conference on Computers and accessibility, pp. 3-10, ACM, 2008.

[3] J. Bishop, "The internet for educating individuals with social impairments," Journal of Computer Assisted Learning, vol. 19, no. 4, pp. 546-556, 2003.

[4] D. Moore, P. McGrath, and J. Thorpe, "Computer-aided learning for people with autism-a framework for research and development," Innovations in Education and Training International, vol. 37, no. 3, pp. 218-228, 2000.

[5] B. Schuller, E. Marchi, S. Baron-Cohen, H. O'Reilly, D. Pigat, P. Robinson, and I. Daves, "The state of play of asc-inclusion: an integrated internet-based environment for social inclusion of children with autism spectrum conditions," arXiv preprint arXiv:1403.5912, 2014.

[6] H. Tanaka, H. Negoro, H. Iwasaka, and S. Nakamura, "Embodied conversational agents for multimodal automated social skills training in people with autism spectrum disorders," PloS one, vol. 12, no. 8, p. e0182151, 2017.

[7] J. Wainer, D. J. Feil-Seifer, D. A. Shell, and M. J. Mataric, "The role of physical embodiment in human-robot interaction," in ROMAN 2006-The 15th IEEE International Symposium on Robot and Human Interactive Communication, pp. 117-122, IEEE, 2006.

[8] B. Roy and D. Bouyssou, Aide multicritère à la décision: méthodes et cas. Economica Paris, 1993.

[9] J. Figueira, S. Greco, and M. Ehrgott, Multiple criteria decision analysis: state of the art surveys, vol. 78. Springer Science \& Business Media, 2005.

[10] H. W. Park, M. Gelsomini, J. J. Lee, T. Zhu, and C. Breazeal, "Backchannel opportunity prediction for 
social robot listeners," in 2017 IEEE International Conference on Robotics and Automation (ICRA), pp. 2308-2314, IEEE, 2017.

[11] Y. Matsuyama, A. Bhardwaj, R. Zhao, O. Romeo, S. Akoju, and J. Cassell, "Socially-aware animated intelligent personal assistant agent," in Proceedings of the 17th Annual Meeting of the Special Interest Group on Discourse and Dialogue, pp. 224-227, 2016.

[12] J. Cassell, T. Bickmore, M. Billinghurst, L. Campbell, K. Chang, H. Vilhjálmsson, and H. Yan, "Embodiment in conversational interfaces: Rea," in Proceedings of the SIGCHI conference on Human Factors in Computing Systems, pp. 520-527, ACM, 1999.

[13] A. Tartaro and J. Cassell, "Using virtual peer technology as an intervention for children with autism," Towards universal usability: designing computer interfaces for diverse user populations, vol. 231, p. 62, 2007.

[14] S. Finkelstein, A. Ogan, C. Vaughn, and J. Cassell, "Alex: A virtual peer that identifies student dialect," in Proc. Workshop on Culturally-aware Technology Enhanced Learning in conjuction with EC-TEL, 2013.

[15] R. Zhao, T. Sinha, A. W. Black, and J. Cassell, "Socially-aware virtual agents: Automatically assessing dyadic rapport from temporal patterns of behavior," in International conference on intelligent virtual agents, pp. 218-233, Springer, 2016.

[16] A. Tartaro and J. Cassell, "Authorable virtual peers for autism spectrum disorders," in Proceedings of the Combined workshop on Language-Enabled Educational Technology and Development and Evaluation for Robust Spoken Dialogue Systems at the 17th European Conference on Artificial Intellegence, 2006.

[17] M. Milne, M. Luerssen, T. Lewis, R. Leibbrandt, and D. Powers, "Embodied conversational agents for education in autism," in A comprehensive Book on Autism Spectrum Disorders, InTech, 2011.

[18] E. Mower, M. P. Black, E. Flores, M. Williams, and S. Narayanan, "Rachel: Design of an emotionally targeted interactive agent for children with autism," in Multimedia and Expo (ICME), 2011 IEEE International Conference on, pp. 1-6, IEEE, 2011.

[19] S. Druga, R. Williams, C. Breazeal, and M. Resnick, "Hey google is it ok if i eat you?: Initial explorations in child-agent interaction," in Proceedings of the 2017 Conference on Interaction Design and Children, pp. 595-600, ACM, 2017.

[20] R. Williams, C. V. Machado, S. Druga, C. Breazeal, and P. Maes, "My doll says it's ok: a study of children's conformity to a talking doll," in Proceedings of the 17th ACM Conference on Interaction Design and Children, pp. 625-631, ACM, 2018.

[21] J.-J. Cabibihan, H. Javed, M. Ang, and S. M. Aljunied, "Why robots? a survey on the roles and benefits of social robots in the therapy of children with autism," International journal of social robotics, vol. 5, no. 4, pp. 593-618, 2013.

[22] B. Scassellati, H. Admoni, and M. Matarić, "Robots for use in autism research," Annual review of biomedical engineering, vol. 14, pp. 275-294, 2012.

[23] J. J. Diehl, L. M. Schmitt, M. Villano, and C. R. Crowell, "The clinical use of robots for individuals with autism spectrum disorders: A critical review," Research in autism spectrum disorders, vol. 6, no. 1, pp. 249-262, 2012.
[24] H. Kozima, M. P. Michalowski, and C. Nakagawa, "Keepon," International Journal of Social Robotics, vol. 1, no. 1, pp. 3-18, 2009.

[25] B. Robins, K. Dautenhahn, and J. Dubowski, "Does appearance matter in the interaction of children with autism with a humanoid robot?," Interaction studies, vol. 7, no. 3, pp. 479-512, 2006.

[26] C. Breazeal et al., "A motivational system for regulating human-robot interaction," in Aaai/iaai, pp. 54-61, 1998.

[27] V. Ferretti and E. Gandino, "Co-designing the solution space for rural regeneration in a new world heritage site: A choice experiments approach," European Journal of Operational Research, vol. 268, no. 3, pp. 1077-1091, 2018.

[28] V. Ferretti, S. Guney, G. Montibeller, and D. von Winterfeldt, "Testing best practices to reduce the overconfidence bias in multi-criteria decision analysis," in 2016 49th Hawaii International Conference on System Sciences (HICSS), pp. 1547-1555, IEEE, 2016.

[29] V. Ferretti, "Exploring multi-methodology approaches for policy support: Case studies and lessons learned," 2016.

[30] S. N. Tani and G. S. Parnell, "Frame the decision opportunity," Handbook of, p. 110, 2013.

[31] R. L. Keeney, "Value-focused thinking: Identifying decision opportunities and creating alternatives," European Journal of operational research, vol. 92, no. 3, pp. 537-549, 1996.

[32] V. Ferretti, "Why is it worth it to expand your set of objectives? impacts from behavioral decision analysis in action.," 2019.

[33] G. S. Parnell, T. A. Bresnick, E. R. Johnson, G. Parnell, T. Bresnick, S. Tani, and E. Johnson, "Craft the decision objectives and value measures," in Handbook of Decision Analysis, pp. 127-148, Wiley, 2013.

[34] R. L. Keeney and R. S. Gregory, "Selecting attributes to measure the achievement of objectives," Operations Research, vol. 53, no. 1, pp. 1-11, 2005.

[35] V. Ferretti and A. Degioanni, "How to support the design and evaluation of redevelopment projects for disused railways? a methodological proposal and key lessons learned," Transportation Research Part D: Transport and Environment, vol. 52, pp. 29-48, 2017.

[36] V. Ferretti, M. Bottero, and G. Mondini, "Decision making and cultural heritage: An application of the multi-attribute value theory for the reuse of historical buildings," Journal of cultural heritage, vol. 15, no. 6, pp. 644-655, 2014.

[37] G. Montibeller and A. Franco, "Decision and risk analysis for the evaluation of strategic options," Supporting strategy: frameworks, methods and models, pp. 251-284, 2007.

[38] V. Belton and T. Stewart, Multiple criteria decision analysis: an integrated approach. Springer Science \& Business Media, 2002. 